\title{
A model for madness?
}

\section{Dream consciousness: our understanding of the neurobiology of sleep offers insight into abnormalities in the waking brain.}

\section{Allan}

Allan Hobson ders. The presence in both of hallucinations and delusions, cognitive abnormalities and emotional intensifications had also been emphasized by earlier, more physiologically oriented psychiatrists, but the psychoanalysts soon eschewed neurobiology and attempted to explain both dreams and psychosis psychodynamically, without reference to the brain. A century of progress in neurobiology and in sleep and dream research now encourages a new look at this old question.

Recent advances in brain imaging, coupled with cellular and molecular neurobiology data, have given us a remarkably clear picture of the differences in brain activity between waking and activated states of sleep, such as rapid eye movement (REM), when intense dreaming most commonly occurs. During waking, consciousness is dominated by externally driven percepts, whereas internally generated images are rare. During REM sleep, the exact opposite rule applies - externally driven percepts are rare, whereas consciousness is dominated by internally generated images. Conversely, descriptions of thinking, which are common in reports of waking consciousness, are rare in reports of dreaming. When the mind is dominated by internal percepts, as it is in dreaming and psychotic states, the capacity to generate thoughts is greatly weakened. Rationality can thus, understandably, fall victim to a change in brain state.

To summarize the extensive data it is useful to look at the results of recent positron emission tomography (PET) scans and brain injury data from humans, and to compare them with earlier results of laboratory experiments in other mammals. The emphasis is on the distinct physiology of REM sleep, but it should be recognized that dreams associated with non-REM sleep are also created by a brain whose physiology has shifted strongly from that of waking towards that of REM.

One of the important causes of dream hallucinations is the general and widespread activation of the brain that occurs in conjunction with a blockade of external sensory inputs and motor outputs in REM sleep. In REM, selective activation of the cortical region responsible for visuospatial integration is seen.

During REM sleep, there is also selective activation of the amygdala and other parts of the limbic system. This is relevant for our

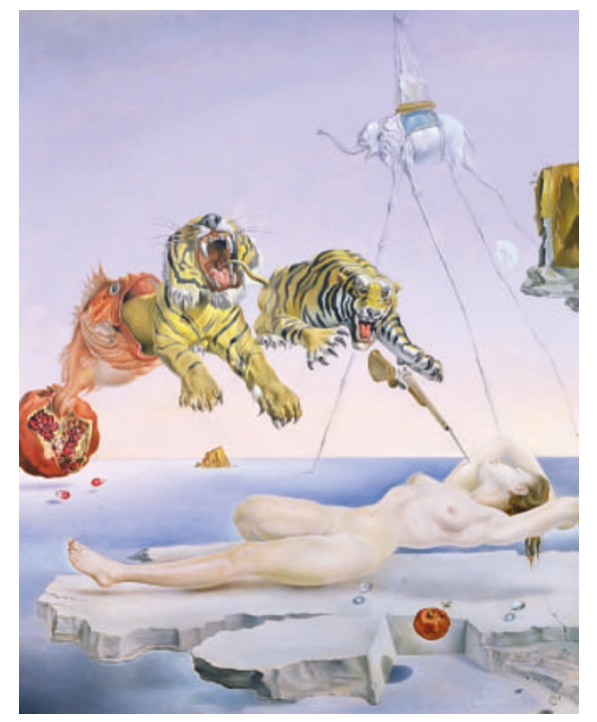

understanding of the heightened emotion especially the feelings of anxiety, anger and elation - that so commonly dictate the development of a dream plot. An exaggeration of emotional activation is also common in mental illness, when it may contribute to impairment of rationality. An idea that helps to unite these findings is that emotion is itself an internally generated percept that powerfully influences conscious experience.

Human PET studies reveal a selective deactivation of the dorsolateral prefrontal cortex in REM state compared with waking. This brain region, which is assumed to be essential to working memory, directed attention and volition, remains inactive during REM. Subjects tell us that in this state they cannot use working memory effectively, are unable to sustain attention and do not will their dream actions. Selective frontal lobe deactivation has also been reported in PET studies of schizophrenia, and may account for the difficulties that these patients have in organizing their thoughts, integrating them with emotion, and translating them into appropriate actions.

Three aminergic neuronal systems — the noradrenergic, the serotonergic and the histaminergic - all shut down in REM. At the same time, the cholinergic system of the pontine tegmentum is activated. Thus, the entire brain is activated but aminergically demodulated except for dopamine (whose release continues at waking levels across all states of sleep). An abnormal sensitivity to dopamine is thought to mediate psychosis and its unmodulated action in REM may contribute to the madness of dreams. As norepinephrine is important for attention and serotonin is critical for learning and memory, it is likely that the decreases in thinking during dreams are due in part to these remarkable and profound changes in brain chemistry.

As well as showing a decline in the capacity to think, formal state studies of dreaming also reveal a decrease in the capacity for orientation (times, places and persons change without notice) and a corresponding increase in the capacity to confabulate (giving the cognitive defects a coherence which is emotionally salient however illogical). Dream bizarreness and the confabulatory quality, coupled with the memory defects and the increase in visual hallucinations, suggests that dreaming may be a functional delirium, much like the psychosis associated with alcohol and drug abuse. Indeed, the delirium-like dreams of normal sleep are produced by changes in the same brain neuromodulatory systems that are affected by drugs en route to clinical delirium.

Now that we have this preliminary, but precise, model for how consciousness is altered by normal and abnormal changes in the state of the brain, we can conduct more hypothesisdriven studies of the brain-mind connection. For example, we can study regional-brain activation in the same animals from which we have learned so much at the cellular and molecular level. In those animals, we can also use drugs to alter a brain state experimentally and predict changes in regional activation pattern. In humans, we can use behavioral techniques as well as drugs to experimentally alter the conscious state of our human subjects; and we can measure predicted changes in regional brain activation with the real-time technique of functional magnetic resonance imaging.

To say that the mind-body problem is solved would be provocative and invite derision, but we have made important progress, and future directions of this approach are now clearly indicated. The mind-body problem can now be confronted by taking advantage of the natural changes in brain-mind state that contrast waking and dreaming.

Allan Hobson is in the Department of Psychiatry, Harvard Medical School, Boston, Massachusetts 02115, USA.

\section{FURTHER READING}

Hobson, J. A. 13 Dreams Freud Never Had: A New Mind Science (Pi Press, New York, 2004).

Hobson, J. A. et al. Behav. Brain Sci. 23, 793-842 (2000). Snyder, S. H. Drugs and the Brain (Scientific American Books, New York, 1986).

Weinberger, D. R. \& McClure, R. K. Arch. Gen. Psych. 59, 553-558 (2002).

Solms, M. The Neuropsychology of Dreams (Lawrence Erlbaum Associates, New York, 1997). 